\title{
An Uniformly Minimum Variance Unbiased Point Estimator Using Fuzzy Observations
}

\author{
Mohammad Ghasem Akbari and Abdolhamid Rezaei \\ Ferdowsi University of Mashhad, Iran
}

\begin{abstract}
This paper proposes a new method for uniformly minimum variance unbiased fuzzy point estimation. For this purpose we make use of a uniformly minimum variance unbiased estimator and we develop this new method for a fuzzy random sample $\tilde{X}_{1}, \ldots, \tilde{X}_{n}$ is induced by $X_{1}, \ldots, X_{n}$ on the same probability space.

Zusammenfassung: In diesem Aufsatz schlagen wir eine neue Methode vor, um einen unverzerrten Fuzzy-Punktschätzer mit gleichmäßig minimaler Varianz zu bekommen. Dazu verwenden wir einen unverzerrten Punkt Schätzer mit gleichmäßig minimaler Varianz und entwickeln diese neue Methode für eine Fuzzy-Zufallsstichprobe $\tilde{X}_{1}, \ldots, \tilde{X}_{n}$, welche aus $X_{1}, \ldots, X_{n}$ auf dem selben Wahrschenlichkeitsraum erzeugt ist.
\end{abstract}

Keywords: Fuzzy Random Variable, Fuzzy Statistic, Signed Distance, Fuzzy Unbiased Estimator, Fuzzy Variance.

\section{Introduction}

Statistical analysis in traditional form is based on crispness of data, random variables (RV's), point estimations, hypotheses, parameters, and so on. But there are many other situations in which the above mentioned concepts are imprecise. The point estimation approaches are frequently used in statistical inference. On the other hand, the theory of fuzzy sets is a well known tool for formulation and analysis of imprecise and subjective concepts. Therefore, the uniformly minimum variance unbiased fuzzy estimator (UMVUFE) with fuzzy data can be important. The problem of point estimation for an unknown parameter, using fuzzy data, is developed in different approaches.

Kruse (1984) and Kruse and Meyer (1987) explained some methods for point and interval estimation for examples of fuzzy random variables (FRV's). Buckley (1983) studied the problem of estimation, with fuzzy data by a fuzzy decision making approach. Okuda (1987) considered fuzzy observations to estimate moments and parameters and he discusses maximum likelihood estimators and the loss of information due to fuzziness. Viertl (1996) studied nonparametric methods in estimation using fuzzy data. Lopez-Diaz and Gil (1998) derived some statistical inference methods, and studied their applications specially in statistical decision theory with fuzzy losses and fuzzy utilities. Cai et al. (1991) proposed a method for estimating parameters of membership functions through defining a likelihood function and studied its applications in fuzzy software reliability modelling. Cai (1993) discussed parameter estimation methods for normal membership functions. Lubiano et al. (1999) and Sadeghpour and Gien (2002) studied a Rao-Blackwell type theorem for FRV's. Lopez-Diaz and Gil (1998) defined a fuzzy unbiased estimator of the sample mean in random sampling with replacement from a finite population. Garcia 
et al. (2001) illustrated estimating the expected value of a FRV in the stratified random sampling. Some methods of statistical inference with fuzzy data are reviewed by Viertl (2002a, 2002b).

There are some researches regarding the Bayesian point estimation methods combined with ideas from fuzzy set theory. Hryniewicz (2002) proposed the notion of fuzzy Bayes point estimation for fuzzy data. Gertner and Zhu (1997), based on two extensions of likelihood function, generalized Bayesian estimates for use when sample information and prior distribution are fuzzy. They applied their method to forest survey. Uemura (1991, 1993) formulated the fuzzy Bayes decision rule to facilitate determination of the loss function of a Bayes decision rule in a fuzzy environment. Wu (2003) studied fuzzy estimators of fuzzy parameters based on FRV's. Finally, Hong-Zhong et al. (2006) proposed a new method to determine the membership function of the parameter estimate of a multi-parameter distribution. This method can be used to determine the membership function of Bayesian estimates of a multi-parameter distribution.

In this paper we organize the matter in the following way. In Section 2 we describe some basic concepts of canonical fuzzy numbers and FRV's. Also, we apply the ranking fuzzy numbers based on signed distance (Yao and $\mathrm{Wu}, 2000$ ) between fuzzy canonical numbers. In Section 3 we summarize the research results report in the literature on uniformly minimum variance unbiased estimators (UMVUE's). Section 4 is devoted to describe UMVUE's using fuzzy data. Finally, some numerical examples are presented in Section 5 in order to illustrate our proposed method.

\section{Preliminaries}

Let $(\Omega, \mathcal{F}, P)$ be a probability space. A RV $X$ is a measurable function from $(\Omega, \mathcal{F}, P)$ to $\left(\mathcal{X}, \mathcal{B}, P_{X}\right)$, where $P_{X}$ is the probability measure induced by $X$ and is called the distribution of $X$, i.e.,

$$
P_{X}(A)=P(X \in A)=\int_{X \in A} d P, \quad \forall A \in \mathcal{B} .
$$

If $P_{X}$ is dominated by a $\sigma$-finite measure $v$, then by the Radon-Nikodym theorem (see Billingsley, 1995) we have

$$
P_{X}(A)=\int_{X \in A} f(x \mid \theta) d v(x), \quad \forall A \in \mathcal{B},
$$

where $f(x \mid \theta)$ is the Radon-Nikodym derivative of $P_{X}$ with respect to $v$ and is called probability density function (PDF) of $X$ with respect to $v$. In a statistical context the measure $v$ is usually a "counting measure" or a "Lebesgue measure", hence $P_{X}(A)$ is calculated by $\sum_{x \in A} f(x \mid \theta)$ or $\int_{A} f(x \mid \theta) d x$, respectively.

Let $S_{X}=\{x \in \mathcal{X} \mid f(x \mid \theta)>0\}$ be the "support" or "sample space" of $X$, then a fuzzy subset $\tilde{x}$ of $S_{X}$ is defined by its membership function $\mu_{\tilde{x}}: S_{X} \rightarrow[0,1]$. We denote by $\tilde{x}_{\alpha}=\left\{x: \mu_{\tilde{x}}(x) \geq \alpha\right\}$ the $\alpha$-cut set of $\tilde{x}$ and $\tilde{x}_{0}$ is the closure of the set $\left\{x: \mu_{\tilde{x}}(x)>0\right\} . \tilde{x}$ is called normal fuzzy set if there exist $x \in S_{X}$ such that $\mu_{\tilde{x}}(x)=1$ and is called convex fuzzy set if $\mu_{\tilde{x}}(\lambda x+(1-\lambda) y) \geq \min \left(\mu_{\tilde{x}}(x), \mu_{\tilde{x}}(y)\right)$ for $\lambda \in[0,1]$. 
The fuzzy set $\tilde{x}$ is called a fuzzy number if $\tilde{x}$ is a normal convex fuzzy set and its $\alpha$-cut set is bounded for all $\alpha \neq 0$. $\tilde{x}$ is called a closed fuzzy number if $\tilde{x}$ is a fuzzy number and its membership function $\mu_{\tilde{x}}$ is upper semicontinous. $\tilde{x}$ is called a bounded fuzzy number if $\tilde{x}$ is a fuzzy number and its membership function $\mu_{\tilde{x}}$ has compact support.

If $\tilde{x}$ is a closed and bounded fuzzy number with $x_{\alpha}^{L}=\inf \left\{x: x \in \tilde{x}_{\alpha}\right\}$ and $x_{\alpha}^{U}=$ $\sup \left\{x: x \in \tilde{x}_{\alpha}\right\}$ and its membership function is strictly increasing on $\left[x_{\alpha}^{L}, x_{1}^{L}\right]$ and strictly decreasing on $\left[x_{1}^{U}, x_{\alpha}^{U}\right]$, then $\tilde{x}$ is called canonical fuzzy number.

Given a real number $x \in S_{X}$, we can induce a fuzzy number $\tilde{x}$ with membership function $\mu_{\tilde{x}}(r)$ such that $\mu_{\tilde{x}}(x)=1$ and $\mu_{\tilde{x}}(r)<1$ for $r \neq x$. Let $X$ be a RV with support $S_{X}$ and $\mathcal{F}\left(S_{X}\right)$ be a set of all fuzzy real numbers induced by the real numbers $S_{X}$. If $\mathcal{F}(\mathcal{R})$ are canonical fuzzy real numbers on $\mathcal{R}$ then it is obvious that $\mathcal{F}\left(S_{X}\right) \subseteq \mathcal{F}(\mathcal{R})$.

Definition 2.1 A FRV is a function $\tilde{X}: \Omega \rightarrow \mathcal{F}\left(S_{X}\right)$, where

$$
\left\{(\omega, x): \omega \in \Omega, x \in \tilde{X}_{\alpha}(\omega)\right\} \in \mathcal{F} \times \mathcal{B}, \quad \forall \alpha \in[0,1],
$$

such that $\tilde{X}$ is induced by $X$.

Lemma 2.1 Let $\mathcal{F}(\mathcal{R})$ be a canonical fuzzy real number system. Then $\tilde{X}$ is a FRV iff $X_{\alpha}^{L}$ and $X_{\alpha}^{U}$ are $R V$ 's for all $\alpha \in[0,1]$.

Definition 2.2 Let $X$ be a $R V$ with cumulative distribution function $(C D F) F(x)$ and let $\tilde{X}$ be a FRV induced by X. The fuzzy function $\tilde{F}(\tilde{x})$ is called fuzzy cumulative distribution function of the FRV $\tilde{X}$, whenever its membership function equals

$$
\mu_{\tilde{F}(\tilde{x})}(y)=\sup _{0 \leq \alpha \leq 1} \alpha I_{\left[F_{\alpha}^{L}(\tilde{x}), F_{\alpha}^{U}(\tilde{x})\right]}(y)
$$

where

$$
\begin{aligned}
& F_{\alpha}^{L}(\tilde{x})=\inf _{\alpha \leq \beta \leq 1}\left\{F(x): x \in\left[x_{\beta}^{L}, x_{\beta}^{U}\right]\right\} \\
& F_{\alpha}^{U}(\tilde{x})=\sup _{\alpha \leq \beta \leq 1}\left\{F(x): x \in\left[x_{\beta}^{L}, x_{\beta}^{U}\right]\right\} .
\end{aligned}
$$

The interval $\left[F_{\alpha}^{L}(\tilde{x}), F_{\alpha}^{U}(\tilde{x})\right]$ contains all of the CDF of each $x_{\beta}^{L}$ and $x_{\beta}^{U}$ for $\beta \geq \alpha$.

To assert the fuzzy expectation of a FRV $\tilde{X}$, we first define $\mathrm{E}_{\alpha}^{L}(\tilde{X})$ and $\mathrm{E}_{\alpha}^{U}(\tilde{X})$ as

$$
\begin{aligned}
& \mathrm{E}_{\alpha}^{L}(\tilde{X})=\inf _{\alpha \leq \beta \leq 1}\left\{\mathrm{E}(X): X \in\left[X_{\beta}^{L}, X_{\beta}^{U}\right]\right\}, \\
& \mathrm{E}_{\alpha}^{U}(\tilde{X})=\sup _{\alpha \leq \beta \leq 1}\left\{\mathrm{E}(X): X \in\left[X_{\beta}^{L}, X_{\beta}^{U}\right]\right\} .
\end{aligned}
$$

The interval $\left[\mathrm{E}_{\alpha}^{L}(\tilde{X}), \mathrm{E}_{\alpha}^{U}(\tilde{X})\right]$ will contain all of the expectations of each $\mathrm{RV} X_{\beta}^{L}$ and $X_{\beta}^{U}$ for $\beta \geq \alpha$.

Definition 2.3 Let $\tilde{X}$ be a FRV induced by X. Then the fuzzy expectation of $\tilde{X}$ is a fuzzy number $\tilde{\mathrm{E}}(\tilde{X})$ with membership function

$$
\mu_{\tilde{\mathrm{E}}(\tilde{X})}(y)=\sup _{0 \leq \alpha \leq 1} \alpha I_{\left[\mathrm{E}_{\alpha}^{L}(\tilde{X}), \mathrm{E}_{\alpha}^{U}(\tilde{X})\right]}(y) .
$$


Several ranking methods have been proposed so far by Cheng (1998), Modarres and Sadi-Nezhad (2001), and Nojavan and Ghazanfari (2006). Here we use another ranking system for canonical fuzzy numbers which is very realistic and is defined by Yao and $\mathrm{Wu}$ (2000) as follows.

Definition 2.4 For any $a, b \in \mathcal{R}$ define their signed distance by $d^{*}(a, b)=a-b$. Thus, we have the following possibilities to define the rank of any two numbers on $\mathcal{R}$ as

$$
\begin{aligned}
& d^{*}(a, b)>0 \Leftrightarrow d^{*}(a, 0)>d^{*}(b, 0) \Leftrightarrow a>b, \\
& d^{*}(a, b)<0 \Leftrightarrow d^{*}(a, 0)<d^{*}(b, 0) \Leftrightarrow a<b, \\
& d^{*}(a, b)=0 \Leftrightarrow d^{*}(a, 0)=d^{*}(b, 0) \Leftrightarrow a=b .
\end{aligned}
$$

Definition 2.5 For each pair $\tilde{a}, \tilde{b} \in \mathcal{F}(\mathcal{R})$ define the signed distance as

$$
d(\tilde{a}, \tilde{b})=\int_{0}^{1}\left(M_{\alpha}(\tilde{a})-M_{\alpha}(\tilde{b})\right) d \alpha=\int_{0}^{1} d^{*}\left(M_{\alpha}(\tilde{a}), M_{\alpha}(\tilde{b})\right) d \alpha,
$$

where $M_{\alpha}(\tilde{a})$ and $M_{\alpha}(\tilde{b})$ equal $\left(a_{\alpha}^{L}+a_{\alpha}^{U}\right) / 2$ and $\left(b_{\alpha}^{L}+b_{\alpha}^{U}\right) / 2$, respectively. Furthermore, $d(\tilde{a}, \tilde{b})$ denotes the distance between $\tilde{a}$ and $\tilde{b}$.

Definition 2.6 (Yao and $W u$, 2000) For each $\tilde{a}, \tilde{b} \in \mathcal{F}(\mathcal{R})$, define their ranking by

$$
\begin{aligned}
& d(\tilde{a}, \tilde{b})>0 \Leftrightarrow d(\tilde{a}, 0)>d(\tilde{b}, 0) \Leftrightarrow \tilde{a} \succ \tilde{b}, \\
& d(\tilde{a}, \tilde{b})<0 \Leftrightarrow d(\tilde{a}, 0)<d(\tilde{b}, 0) \Leftrightarrow \tilde{a} \prec \tilde{b}, \\
& d(\tilde{a}, \tilde{b})=0 \Leftrightarrow d(\tilde{a}, 0)=d(\tilde{b}, 0) \Leftrightarrow \tilde{a} \approx \tilde{b} .
\end{aligned}
$$

\section{The UMVUE with Crisp Data}

In this section, we describe concepts of UMVUE's with crisp data. Let $X_{1}, \ldots, X_{n}$ be a random sample of size $n$, where the $X_{i}$ 's have PDF $f(x \mid \theta)$ with unknown parameter $\theta, \theta \in \Theta$, and $x_{1}, \ldots, x_{n}$ are realizations of $X_{1}, \ldots, X_{n}$, respectively. Recall that an estimator $T\left(X_{1}, \ldots, X_{n}\right)$ of $\theta$ is unbiased iff $\mathrm{E}\left[T\left(X_{1}, \ldots, X_{n}\right)\right]=\theta$ for any $\theta \in \Theta$. If an unbiased estimator of $\theta$ exists, then $\theta$ is called an estimable parameter.

Definition 3.1 An unbiased estimator $T\left(X_{1}, \ldots, X_{n}\right)$ of $\theta$ is called uniformly minimum variance unbiased estimator $(U M V U E)$ iff

$$
\operatorname{var}\left[\left(T\left(X_{1}, \ldots, X_{n}\right)\right)\right] \leq \operatorname{var}\left[\left(U\left(X_{1}, \ldots, X_{n}\right)\right],\right.
$$

for any $\theta \in \Theta$ and any other unbiased estimator $U\left(X_{1}, \ldots, X_{n}\right)$ of $\theta$.

The derivation of such an UMVUE is relatively simple if there exist a complete sufficient statistic for $\theta \in \Theta$.

Theorem 3.1 (Lehmann-Scheffé) Suppose that there exists a complete sufficient statistic $T\left(X_{1}, \ldots, X_{n}\right)$ for $\theta \in \Theta$. If $\theta$ is estimable, then there exists an unique unbiased estimator of $g(\theta)$ of the form $h(T)$ with a Borel function $h$. Furthermore, $h(T)$ is the unique UMVUE of $\theta$. 
There are two typically ways to derive a UMVUE when a complete sufficient statistic is available. The first one is to solve for $h$ when the distribution of $T$ is available. The second method to derive an UMVUE when there exists a complete sufficient statistic (used here), is to condition on $T$, i.e., if $U\left(X_{1}, \ldots, X_{n}\right)$ is an unbiased estimator of $\theta$, then $\mathrm{E}\left[\left(U\left(X_{1}, \ldots, X_{n}\right) \mid T\right)\right]$ is the UMVUE of $\theta$. To apply this method, we do not need the distribution of $T$ but need to work out the conditional expectation $\mathrm{E}\left[U\left(X_{1}, \ldots, X_{n} \mid T\right)\right]$. From the uniqueness of the UMVUE, it does not matter which $U\left(X_{1}, \ldots, X_{n}\right)$ is used. We should choose $U\left(X_{1}, \ldots, X_{n}\right)$ to make the calculation of $\mathrm{E}\left[U\left(X_{1}, \ldots, X_{n}\right) \mid T\right]$ as easy as possible. For a review in more details, see Shao (2003).

\section{An UMVUE with Fuzzy Data}

Now we introduce concepts of an UMVUFE.

Definition 4.1 Let $\tilde{X}$ and $\tilde{Y}$ be two FRV's induced by $X$ and $Y$. We say that $\tilde{X}$ and $\tilde{Y}$ are independent iff each $R V$ in the set $\left\{X_{\alpha}^{L}, X_{\alpha}^{U}: 0 \leq \alpha \leq 1\right\}$ is independent of each $R V$ in the set $\left\{Y_{\alpha}^{L}, Y_{\alpha}^{U}: 0 \leq \alpha \leq 1\right\}$.

Definition 4.2 We say that $\tilde{X}$ and $\tilde{Y}$ are identically distributed iff $X_{\alpha}^{L}, Y_{\alpha}^{L}$ are identically distributed, and $X_{\alpha}^{U}, Y_{\alpha}^{U}$ are identically distributed for all $\alpha \in[0,1]$.

Let $\tilde{\mathbf{X}}=\left(\tilde{X}_{1}, \ldots, \tilde{X}_{n}\right)$ be a fuzzy random sample induced by $\mathbf{X}=\left(X_{1}, \ldots, X_{n}\right)$ on the sample probability space $(\Omega, \mathcal{F}, P)$, and with membership functions $\mu_{\tilde{X}_{i}}(y)$.

In traditional statistics, parameter estimates are functions of the sample. Formally, based on the sample $\mathbf{X}$ for a RV $X$ with sample space $S_{X}$, PDF $f(x \mid \theta)$ and parameter space $\Theta$, estimators are functions $\delta(\mathbf{X})$ defined on the sample space $S_{X_{1}} \times \cdots \times S_{X_{n}}$. For a realized sample $\mathbf{x}=\left(x_{1}, \ldots, x_{n}\right)$ an estimation $\widehat{\theta}$ of the parameter $\theta$ is obtained by $\delta(\mathbf{x})=\widehat{\theta} \in \Theta$.

For a fuzzy random sample $\tilde{\mathbf{X}}$ induced by $\mathbf{X}$ with membership functions $\mu_{\tilde{X}_{i}}(y)$ the function $\tilde{\delta}(\tilde{\mathbf{x}})$, where $\tilde{\mathbf{x}}=\left(\tilde{x}_{1}, \ldots, \tilde{x}_{n}\right)$, becomes a fuzzy element $\widehat{\tilde{\theta}} \in \Theta$, whose membership function $\mu_{\tilde{\delta}(x)}$ is derived by applying the extension principle (Klir and Yuan, 1995), i.e.

$$
\mu_{\tilde{\delta}(\tilde{\mathbf{x}})}(d)= \begin{cases}\sup \left\{\min _{1 \leq i \leq n} \mu_{\tilde{X}_{i}}\left(x_{i}\right): \delta\left(x_{1}, \ldots, x_{n}\right)=d\right\}, & \delta^{-1}(d) \neq \emptyset \\ 0, & \delta^{-1}(d)=\emptyset\end{cases}
$$

for any $d \in \Theta$.

For a fuzzy random sample $\tilde{\mathbf{X}}$, the fuzzy number $\tilde{\delta}(\tilde{\mathbf{X}})$ is called a fuzzy statistic, provided that it is not a function of any unknown parameter. Let $\tilde{X}_{i}$ and $X_{i}$ have CDF $\tilde{F}$ and $F$, respectively. A fuzzy statistic $\tilde{\delta}(\tilde{\mathbf{X}})$ is said to be a (point) estimator of $\tilde{\theta}$, if

$$
\tilde{\delta}: \mathcal{F}\left(S_{X_{1}}\right) \times \cdots \times \mathcal{F}\left(S_{X_{n}}\right) \rightarrow \Theta
$$

where $\mathcal{F}\left(S_{X_{i}}\right)$ is the space of fuzzy values of $\tilde{X}_{i}$. 
Each $\alpha$-cut set of $\tilde{\delta}(\tilde{\mathbf{X}})$ depends on $\mathbf{X}$. Suppose $\left\{\delta(\mathbf{X}): X_{i} \in\left(\tilde{X}_{i}\right)_{\alpha}\right\}$ is a crisp estimator of $\theta$ that is obtained by $\alpha$-cut of $\tilde{\delta}(\tilde{\mathbf{X}})$. Define

$$
\begin{aligned}
\delta_{\alpha}^{L} & =\delta_{\alpha}^{L}(\mathbf{X})=\inf _{\alpha \leq \beta \leq 1}\left\{\delta(\mathbf{X}): X_{i} \in\left(\tilde{X}_{i}\right)_{\beta}\right\}, \\
\delta_{\alpha}^{U} & =\delta_{\alpha}^{U}(\mathbf{X})=\sup _{\alpha \leq \beta \leq 1}\left\{\delta(\mathbf{X}): X_{i} \in\left(\tilde{X}_{i}\right)_{\beta}\right\},
\end{aligned}
$$

where the interval $I\left(\delta_{\alpha}\right)=\left[\delta_{\alpha}^{L}, \delta_{\alpha}^{U}\right]$ contains all the crisp estimators of each RV $X_{i \beta}^{L}$ and $X_{i \beta}^{U}$ for $\beta \geq \alpha$.

Definition 4.3 The fuzzy estimator $\tilde{\delta}(\tilde{\mathbf{X}})$ is unbiased for $\tilde{\theta}$ iff for all $\alpha \in[0,1]$ and $\delta_{0} \in$ $\left[\delta_{\alpha}^{L}, \delta_{\alpha}^{U}\right]$ there exists a $\theta_{0} \in \tilde{\theta}_{\alpha}$ such that $\mathrm{E}\left(\delta_{0}\right)=\theta_{0}$.

Let $\tilde{\mathcal{D}}$ be a nonempty set of all fuzzy unbiased estimators $\tilde{\delta}(\tilde{\mathbf{X}})$ for $\tilde{\theta}$.

Definition 4.4 The fuzzy estimator $\tilde{\delta}(\tilde{\mathbf{X}})$ is sufficient for $\tilde{\theta}$ iff for each $\alpha \in[0,1]$ and $\delta \in I\left(\delta_{\alpha}\right), \delta$ is a sufficient statistics for $\theta \in \tilde{\theta}_{\alpha}$.

Now we define the membership function of the fuzzy variance function of $\tilde{\delta}(\tilde{\mathbf{X}})$, denoted by $\widetilde{\operatorname{var}}(\tilde{\delta}(\tilde{\mathbf{X}}))$, as

$$
\mu_{\widetilde{\mathrm{var}}}(y)=\sup _{0 \leq \alpha \leq 1} \alpha I_{\left[\operatorname{var}_{\alpha}^{L}, \operatorname{var}_{\alpha}^{U}\right]}(y)
$$

where

$$
\operatorname{var}_{\alpha}^{L}=\inf _{\delta \in I\left(\delta_{\alpha}\right)}\{\operatorname{var}(\delta(\mathbf{X}))\}, \quad \operatorname{var}_{\alpha}^{U}=\sup _{\delta \in I\left(\delta_{\alpha}\right)}\{\operatorname{var}(\delta(\mathbf{X}))\}
$$

Definition 4.5 $\tilde{\delta^{*}}(\tilde{\mathbf{X}})$ is an UMVUFE of $\tilde{\theta}$ iff $\tilde{\delta}$ is unbiased and

$$
\widetilde{\operatorname{var}^{*}}=\widetilde{\operatorname{var}}\left(\tilde{\delta^{*}}\right) \precsim \widetilde{\operatorname{var}}=\widetilde{\operatorname{var}}(\tilde{\delta}), \quad \forall \tilde{\delta} \in \tilde{\mathcal{D}},
$$

where notation " $\approx$ " denotes signed distance.

Theorem 4.1 Let $\mathbf{X}=\left(X_{1}, \ldots, X_{n}\right)$ be a random sample and $\tilde{\mathbf{X}}=\left(\tilde{X}_{1}, \ldots, \tilde{X}_{n}\right)$ be a fuzzy random sample induced by $\mathbf{X}$. If $\tilde{U}(\tilde{\mathbf{X}})$ is a fuzzy unbiased estimator for $\tilde{\theta}$ and $\tilde{T}(\tilde{\mathbf{X}})$ a fuzzy sufficient statistics for $\tilde{\theta}$, then the UMVUFE $\tilde{\delta^{*}}(\tilde{\mathbf{X}})$ has membership function

$$
\mu_{\tilde{\delta^{*}}(\tilde{\mathbf{X}})}(y)=\sup _{0 \leq \alpha \leq 1} \alpha I_{\left[\mathrm{E}_{\alpha}^{L}, \mathrm{E}_{\alpha}^{U}\right]}(y)
$$

in which

$$
\begin{aligned}
\mathrm{E}_{\alpha}^{L} & =\inf _{\alpha \leq \beta \leq 1}\left\{\mathrm{E}[U(\mathbf{X}) \mid T(\mathbf{X})]: X_{i} \in\left(\tilde{X}_{i}\right)_{\beta}\right\}, \\
\mathrm{E}_{\alpha}^{U} & =\sup _{\alpha \leq \beta \leq 1}\left\{\mathrm{E}[U(\mathbf{X}) \mid T(\mathbf{X})]: X_{i} \in\left(\tilde{X}_{i}\right)_{\beta}\right\} .
\end{aligned}
$$

Proof: We first prove that $\tilde{\delta^{*}}$ is a canonical fuzzy number. Define $S_{\alpha}=\left\{\left(x_{1}, \ldots, x_{n}\right)\right.$ : $\left.x_{i} \in\left(\tilde{x}_{i}\right)_{\alpha}\right\}$ and $k\left(x_{1}, \ldots, x_{n}\right)=\mathrm{E}[U(\mathbf{X}) \mid T(\mathbf{X})]$ for $\left(x_{1}, \ldots, x_{n}\right) \in S_{\alpha}$ and $\alpha \in[0,1]$.

We know that $\tilde{x}_{i}$ 's are canonical fuzzy numbers, $k$ is continuous, $S_{\alpha}$ is connected, closed, and bounded implying that the range of $k$ is a closed and bounded interval of real 
numbers. Define $\tilde{\delta_{\alpha}^{*}}=k\left(S_{\alpha}\right)$ for $\alpha \in[0,1]$. Furthermore $\tilde{\delta}_{1}^{*}=\mathrm{E}[U(\mathbf{X}) \mid T(\mathbf{X})] \neq \emptyset$. Hence, $\tilde{\delta}_{\alpha}^{*}$ is a canonical fuzzy number.

To proof unbiasedness, let $\alpha \in[0,1]$ and $\delta_{\alpha}^{*} \in\left[\delta_{\alpha}^{* L}, \delta_{\alpha}^{* U}\right]$. Thus there exists a $X_{i} \in \tilde{X}_{i \alpha}$ such that $\delta_{\alpha}^{*}(\mathbf{X})=\mathrm{E}[U(\mathbf{X}) \mid T(\mathbf{x})], \mathrm{E}\left[\delta_{\alpha}^{*}(\mathbf{X})\right]=\mathrm{E}[U(\mathbf{X})]$. Now we know that $\tilde{U}(\tilde{\mathbf{X}})$ is an unbiased fuzzy estimator. By definition 4.3 there exists a $\theta_{0} \in \tilde{\theta}_{\alpha}$ such that $\mathrm{E}\left[\delta_{\alpha}^{*}(\mathbf{X})\right]=$ $\mathrm{E}[U(\mathbf{X})]=\theta_{0}$. As a result, $\tilde{\delta^{*}}(\tilde{\mathbf{X}})$ is a fuzzy unbiased estimator.

According to definition $4.4, T_{\alpha}$ is a sufficient statistic for $\theta_{0}$ and in the other words, $U(\mathbf{X})$ is unbiased for $\theta_{0}$. Thus

$$
\operatorname{var}\left(\delta_{\alpha}^{*}(\mathbf{X})\right) \leq \operatorname{var}(U(\mathbf{X})) .
$$

According to (1) we have

$$
\operatorname{var}_{\alpha}^{L}(\tilde{U}(\mathbf{X}))=\inf _{U \in I\left(U_{\alpha}\right)}\{\operatorname{var}(U(\mathbf{X}))\}
$$

and for $\tilde{\delta}^{*}(\tilde{\mathbf{X}})$ we can write

$$
\operatorname{var}_{\alpha}^{L}\left(\tilde{\delta}^{*}(\mathbf{X})\right)=\inf _{\delta^{*} \in\left[\mathrm{E}_{\alpha}^{L}, \mathrm{E}_{\alpha}^{U}\right]}\left\{\operatorname{var}\left(\delta^{*}\right)\right\}
$$

Thus, based on (2) we have

$$
\operatorname{var}_{\alpha}^{L}\left(\tilde{\delta}^{*}\right) \leq \operatorname{var}_{\alpha}^{L}(\tilde{U})
$$

and similarly it can be also shown that $\operatorname{var}_{\alpha}^{U}\left(\tilde{\delta}^{*}\right) \leq \operatorname{var}_{\alpha}^{U}(\tilde{U})$. According to the function $M$ in the Yao-Wu signed distance, we have

$$
d\left(\widetilde{\operatorname{var}}^{*}, \widetilde{\operatorname{var}}\right)=\int_{0}^{1}\left(M_{\alpha}\left(\widetilde{\operatorname{var}}^{*}\right)-M_{\alpha}(\widetilde{\operatorname{var}})\right) d \alpha \leq 0,
$$

because $M_{\alpha}\left(\widetilde{\operatorname{var}}^{*}\right) \leq M_{\alpha}(\widetilde{\operatorname{var}})$. Hence, $\widetilde{\operatorname{var}}\left(\tilde{\delta^{*}}(\tilde{\mathbf{X}})\right) \lesssim \widetilde{\operatorname{var}}(\tilde{U}(\tilde{\mathbf{X}}))$.

\section{Numerical Examples}

Now we illustrate the proposed approach for some distributions.

Example 5.1 Let $X$ be a RV from a $N(\theta, 1)$ population, i.e.

$$
f(x \mid \theta)=\frac{1}{\sqrt{2 \pi}} \exp \left(-\frac{1}{2}(x-\theta)^{2}\right), \quad x, \theta \in \mathcal{R} .
$$

Consider $\tilde{x}_{1}, \ldots, \tilde{x}_{n}$ with triangular membership functions $\left(x_{i}-a, x_{i}, x_{i}+b\right)$ given by

$$
\mu_{\tilde{x}_{i}}(y)= \begin{cases}\left(y-x_{i}+a\right) / a, & x_{i}-a \leq y \leq x_{i} \\ \left(x_{i}+b-y\right) / b, & x_{i} \leq y \leq x_{i}+b\end{cases}
$$

for each $1 \leq i \leq n$ and $a, b \geq 0$. We can interpret the canonical fuzzy numbers $\tilde{x}_{i}$ as the values of "near to $x_{i}$ ". We have

$$
\mu_{\tilde{\delta^{*}}(\tilde{\mathbf{X}})}(y)=\sup _{0 \leq \alpha \leq 1} \alpha I_{\left[\mathrm{E}_{\alpha}^{L}, \mathrm{E}_{\alpha}^{U]}\right.}(y),
$$


such that

$$
\mathrm{E}_{\alpha}^{L}=\bar{x}-(1-\alpha) a, \quad \mathrm{E}_{\alpha}^{U}=\bar{x}+(1-\alpha) b,
$$

and we note that in crisp form for this example the UMVUE is $\delta^{*}(\mathbf{X})=\overline{\mathbf{X}}$ and also $\mu_{\tilde{\delta}^{*}(\tilde{\mathbf{x}})}(\bar{x})=1$. For instance when $a=b=5$ and our fuzzy observations $\tilde{x}_{1}, \ldots, \tilde{x}_{6}$ have membership functions

$$
\begin{array}{cc}
\mu_{\tilde{x}_{1}}(y)=\left\{\begin{array}{ll}
(y-40) / 5, & 40 \leq y \leq 45 \\
(50-y) / 5, & 45 \leq y \leq 50
\end{array},\right. & \mu_{\tilde{x}_{2}}(y)= \begin{cases}(y-50) / 5, & 50 \leq y \leq 55 \\
(60-y) / 5, & 55 \leq y \leq 60\end{cases} \\
\mu_{\tilde{x}_{3}}(y)=\left\{\begin{array}{ll}
(y-65) / 5, & 65 \leq y \leq 70 \\
(75-y) / 5, & 70 \leq y \leq 75
\end{array},\right. & \mu_{\tilde{x}_{4}}(y)= \begin{cases}(y-80) / 5, & 80 \leq y \leq 85 \\
(90-y) / 5, & 85 \leq y \leq 90\end{cases} \\
\mu_{\tilde{x}_{5}}(y)=\left\{\begin{array}{ll}
(y-110) / 5, & 110 \leq y \leq 115 \\
(120-y) / 5, & 115 \leq y \leq 120
\end{array},\right. & \mu_{\tilde{x}_{6}}(y)= \begin{cases}(y-117) / 5, & 117 \leq y \leq 122 \\
(127-y) / 5, & 122 \leq y \leq 127 .\end{cases}
\end{array}
$$

We derive

$$
\left[\mathrm{E}_{\alpha}^{L}, \mathrm{E}_{\alpha}^{U}\right]=[82-5(1-\alpha), 82+5(1-\alpha)] .
$$

Hence, $\tilde{\delta^{*}}(\tilde{x})$ is a canonical fuzzy number and $\mu_{\tilde{\delta^{*}}(\tilde{\mathbf{x}})}(82)=1$.

Example 5.2 Let $X$ be a RV from a $\mathrm{E}(\theta, 1)$ population, i.e.,

$$
f(x \mid \theta)=\exp (-(x-\theta)), \quad x>\theta, \theta>0,
$$

and let $\tilde{x}_{i}$ be some fuzzy observations with membership functions

$$
\mu_{\tilde{x}_{i}}(y)= \begin{cases}\exp \left(-\left(y-x_{i}\right)^{2}\right), & x_{i}-0.5 \leq y \leq x_{i}+0.5 \\ 0, & \text { otherwise }\end{cases}
$$

for each $1 \leq i \leq n$. We can interpret the canonical fuzzy numbers $\tilde{x}_{i}$ as the values of "near to $x_{i}$ ". We have

$$
\mu_{\tilde{\delta}^{*}(\tilde{\mathbf{x}})}(y)=\sup _{0 \leq \alpha \leq 1} \alpha I_{\left[\mathrm{E}_{\alpha}^{L}, \mathrm{E}_{\alpha}^{U]}\right.}(y)
$$

such that

$$
\begin{aligned}
& \mathrm{E}_{\alpha}^{L}=\left\{\begin{array}{ll}
x_{(1)}-1 / n-0.5, & 0 \leq \alpha \leq \exp (-0.25) \\
x_{(1)}-1 / n-\sqrt{-\log \alpha}, & \exp (-0.25) \leq \alpha \leq 1
\end{array},\right. \\
& \mathrm{E}_{\alpha}^{U}=\left\{\begin{array}{ll}
x_{(1)}-1 / n+0.5, & 0 \leq \alpha \leq \exp (-0.25) \\
x_{(1)}-1 / n+\sqrt{-\log \alpha}, & \exp (-0.25) \leq \alpha \leq 1
\end{array} .\right.
\end{aligned}
$$

We note that in crisp form for this example the UMVUE is $\delta^{*}(\mathbf{X})=X_{(1)}-1 / n$ and also $\mu_{\tilde{\delta^{*}(\tilde{\mathbf{x}})}}\left(x_{(1)}-1 / n\right)=1$.

Example 5.3 Let $X$ be a RV from a $U \sim(\theta, \theta+1)$ population i.e.,

$$
f(x \mid \theta)=1, \quad \theta \leq x \leq \theta+1,0<\theta<1,
$$

and $\tilde{x}_{i}$ are fuzzy observations with triangular membership functions $\left(x_{i}-a, x_{i}, x_{i}+b\right)$. We have

$$
\mathrm{E}_{\alpha}^{L}=\left(x_{(1)}+x_{(2)}-1\right) / 2-(1-\alpha) a, \quad \mathrm{E}_{\alpha}^{U}=\left(x_{(1)}+x_{(2)}-1\right) / 2+(1-\alpha) b .
$$


Thus, the membership function of $\tilde{\delta^{*}}(\tilde{\mathbf{x}})$ is

$$
\mu_{\tilde{\delta^{*}}(\tilde{\mathbf{x}})}(y)=\sup _{0 \leq \alpha \leq 1} \alpha I_{\left[\mathrm{E}_{\alpha}^{L}, \mathrm{E}_{\alpha}^{U]}\right.}(y) .
$$

Example 5.4 Let $X$ be a RV from an exponential population with mean $1 / \theta$, i.e.,

$$
f(x \mid \theta)=\theta \exp (-\theta x), \quad x>0, \theta>0,
$$

and let $\tilde{x}_{i}$ 's be some fuzzy observations with membership functions

$$
\mu_{\tilde{x}_{i}}(x)= \begin{cases}\exp \left(-\left(x-x_{i}\right)^{2}\right), & x_{i}-0.5 \leq x \leq x_{i}+0.5 \\ 0, & \text { otherwise }\end{cases}
$$

for each $1 \leq i \leq n$. Then we have

$$
\begin{gathered}
\mathrm{E}_{\alpha}^{L}= \begin{cases}\frac{n-1}{n(\bar{x}+0.5)}, & 0 \leq \alpha \leq \exp (-0.25) \\
\frac{n-1}{n(\bar{x}+\sqrt{-\log \alpha})}, & \exp (-0.25) \leq \alpha \leq 1\end{cases} \\
\mathrm{E}_{\alpha}^{U}= \begin{cases}\frac{n-1}{n(\bar{x}-0.5)}, & 0 \leq \alpha \leq \exp (-0.25) \\
\frac{n-1}{n(\bar{x}-\sqrt{-\log \alpha})}, & \exp (-0.25) \leq \alpha \leq 1\end{cases}
\end{gathered}
$$

Thus, the membership function of $\tilde{\delta^{*}}(\tilde{\mathbf{x}})$ is

$$
\mu_{\tilde{\delta^{*}}(\tilde{\mathbf{x}})}(y)=\sup _{0 \leq \alpha \leq 1} \alpha I_{\left[\mathrm{E}_{\alpha}^{L}, \mathrm{E}_{\alpha}^{U]}\right]}(y)
$$

Example 5.5 Let $X$ be a RV with PDF

$$
f(x \mid \theta)=\frac{1}{\theta}, \quad 0<x<\theta .
$$

Let $\tilde{x}_{1}$ and $\tilde{x}_{2}$ be two fuzzy canonical numbers with triangular membership functions $\left(x_{i}-a^{\prime}, x_{i}, x_{i}+b^{\prime}\right)$, then we have

$$
\left[\mathrm{E}_{\alpha}^{L}, \mathrm{E}_{\alpha}^{U}\right]=\left[\frac{n+1}{n}\left(x_{(1)}-(1-\alpha) a^{\prime}\right), \frac{n+1}{n}\left(x_{(1)}+(1-\alpha) b^{\prime}\right)\right] .
$$

Furthermore, the parameter estimate at any $\alpha$-cut level can be calculated with respect to membership function

$$
\mu_{\tilde{\delta^{*}}(\tilde{\mathbf{x}})}(y)=\sup _{0 \leq \alpha \leq 1} \alpha I_{\left[\mathrm{E}_{\alpha}^{L}, \mathrm{E}_{\alpha}^{U]}\right.}(y)
$$

\section{Conclusion}

From the above discussion, it is clear that under Yao-Wu signed distance, the UMVUF estimator propose a canonical fuzzy number. The UMVUF estimator has the smallest fuzzy variance of the other fuzzy estimator for fuzzy parameter $\tilde{\theta}$. 


\section{Acknowledgements}

The authors thank the referees for their comments which helped us to improve the paper.

\section{References}

Billingsley, P.(1995). Probability and Measure (2nd ed.). New York: John Wiley.

Buckley, J. J. (1983). Fuzzy decision making with data: applications to statistics. Fuzzy Sets and Systems, 16, 139-174.

Cai, K. Y. (1993). Parameter estimation of normal fuzzy variables. Fuzzy Sets and Systems, 55, 179-185.

Cai, K. Y., Wen, C. Y., and Zhang, M. L.(1991). Fuzzy variable as a basis for a theory of fuzzy reliability in the possibility context. Fuzzy Sets and Systems, 42, 145-172.

Cheng, C.(1998). A new approach for ranking fuzzy numbers by distance method. Fuzzy Sets and Systems, 95, 307-317.

Garcia, D., Lubiano, M. A., and Alonso, C. (2001). Estimating the expected value of fuzzy random variables in the stratified random sampling from finite populations. Information Sciences, 138, 165-184.

Gertner, G. Z., and Zhu, H.(1997). Bayesian estimation in forest survey when samples or prior information are fuzzy. Fuzzy Sets and Systems, 77, 277-290.

Hong-Zhong, H., Ming, J. Z., and Zhan-Quan, S.(2006). Bayesian reliability analysis for fuzzy lifetime data. Fuzzy Sets and Systems, 157, 1674-1686.

Hryniewicz, O.(2002). Possibilities approach to the Bayes statistical decisions. In P. Grzegorzewski, O. Hryniewicz, and M. A. Gil (Eds.), Soft Methods in Probability, Statistics and Data Analysis (p. 207-218). Heidelberg-New York: Physica Verlag.

Klir, G., and Yuan, B. (1995). Fuzzy sets and fuzzy logic-theory and applications. Upper Saddle River, NJ: Prentice-Hall.

Kruse, R. (1984). Statistical estimation with linguistic data. Information Science, 33, 197-207.

Kruse, R., and Meyer, K. D. (1987). Statistics with Vague Data (Vol. 33). Dordrecht: Reidel.

Lopez-Diaz, M., and Gil, M. A. (1998). Reversing the order of integration in iterated expectations of fuzzy random variables, and statistical applications. Jornal of Statistical Planning and Inference, 74, 11-29.

Lubiano, M. A., Gil, M. A., and Lopez-Diaz, M. (1999). On the Rao-Blackwell theorem for fuzzy random variables. Kybernetica, 35, 167-175.

Modarres, M., and Sadi-Nezhad, S. (2001). Ranking fuzzy numbers by preference ratio. Fuzzy Sets and Systems, 118, 429-436.

Nojavan, M., and Ghazanfari, M. (2006). A fuzzy ranking method by desirability index. Journal of Intelligent and Fuzzy Systems, 17, 27-34.

Okuda, T. (1987). A statistical treatment of fuzzy observations: estimation problems. In Preprints of the 2nd IFSA Congress (p. 51-55).

Sadeghpour, G. B., and Gien, D. (2002). $d_{p, q}$-distance and Rao-Blackwell theorem for fuzzy random variables. In Proceedings of the 8th international Conference of Fuzzy Theory and Technology. Durham, USA. 
Shao, J.(2003). Mathematical Statistics (2nd ed.). New York: Springer-Verlag.

Uemura, Y. (1991). A decision rule on fuzzy events. Japanese Journal of Fuzzy Theory and Systems, 3, 291-300.

Uemura, Y. (1993). A decision rule on fuzzy events under an observation. Journal of Fuzzy Mathematics, 1, 39-52.

Viertl, R.(1996). Statistical Methods for Non-Precise Data. Boca Raton: CRC Press.

Viertl, R.(2002a). Statistics with one-dimensional fuzzy data. In C. B. et al. (Ed.), Statistical Modeling, Analysis and Management of Fuzzy Data (p. 199-212). Heidelberg: Physica-Verlag.

Viertl, R. (2002b). Statistical inference with non-precise data. In Encyclopedia of Life Support Systems. Paris: UNESCO.

$\mathrm{Wu}, \mathrm{H}$. C. (2003). The fuzzy estimators of fuzzy parameters based on fuzzy random variables. European Journal of Operation Research, 146, 101-114.

Yao, J. S., and Wu, K.(2000). Ranking fuzzy numbers based on decomposition principle and signed distance. Fuzzy Sets and Systems, 11, 275-288.

Authors' Address:

Mohammad Ghasem Akbari and Abdolhamid Rezaei

Department of Statistics

School of Mathematical Sciences

Ferdowsi University of Mashhad

91775-1159, Mashhad

Iran

E-mail: g_z_akbari@yahoo.com 